\title{
A direct computer control concept for mammalian cell fermentation processes
}

\author{
Heino Büntemeyer, Rainer Marzahl and Jürgen Lehmann \\ Institute of Cell Culture Technology, University of Bielefeld, P.O. Box 100131,33501 Bielefeld, Germany
}

Key words: Bioreactor, control, computer, fermentation, perfusion

\begin{abstract}
In the last 10 years, new assignments and the special demands of mammalian cells to the culture conditions caused the develoepment of complex small scale fermentation setups. The use of continuous fermentation and cell retention devices requires appropriate process control systems. An arrangement for control and data-acquisition of complex laboratory-scale bioreactors is presented. The fundamental idea was the usage of a standard personal computer, which is connected to pumps, valves and sensors via ADA-transformation. The possibility of free programming allowed the development of user-oriented software, especially designed for the far-reaching requirements of a university laboratory in the field of animal cell culture. Control of aeration, pumps, data-acquisition and data-storage are combined within one program, which allows the automation of standard operations like measurement of $\mathrm{k}_{\mathrm{L}} \mathrm{a}$ - or OTR-values. Pump control algorithms for all common fermentation strategies (batch, fed batch, chemostat, perfusion) are included and can be selected any time during cultivation. Oxygen partial pressure and $\mathrm{pH}$ are controlled via direct digital control ( $\mathrm{ddc}$ ), providing simple adaption of control parameters and set points to current fermentation conditions.
\end{abstract}

\section{Introduction}

\section{Starting conditions}

The comparison between eucaryotic and prokaryotic cells shows, that the former are much more pretentious referred to the fermentation conditions. Adapted to the lower growth rate, the higher susceptibility to shear stress and the higher substrate requirements, special fermentation processes have been developed in the last years. They allow continuous cultivation and include internal or external cell retention systems, like spin filters, continuous centrifuges or hollow fibre membranes. In these sophisticated setups, controlling of pumps for the various medium streams is more complex compared to fed batch or chemostat fermentations.

A process control system (PCS) for common laboratory scale fermenter setups like those, is usually available as a combination of several independent components. Subsystems, controlling aeration, pumps and data acquisition must be combined to provide the possibility of an integrated process control. Information exchange and manipulation of the running process need to take place at different modules of the fermentation setup. This decreases the effectiveness and increases the working effort of mammalian cell cultivation processes.

\section{Demands to a process control system}

There are several aspects that are important for the development of a PCS. First, it must control all process relevant variables, like $\mathrm{pH}$, oxygen partial pressure, liquid flows, etc. Second, the possibility of rational user information and interaction with the system must be provided. Finally, the data acquisition should be performed in such way, that the stored data can be processed by word processing or spreadsheet software.

Common PCS have been laid out for a wide spectrum of even prokaryotic fermentations. Thus, only a few functions, specifically used for mammalian cell cultivation, are implemented. Others must be added. 


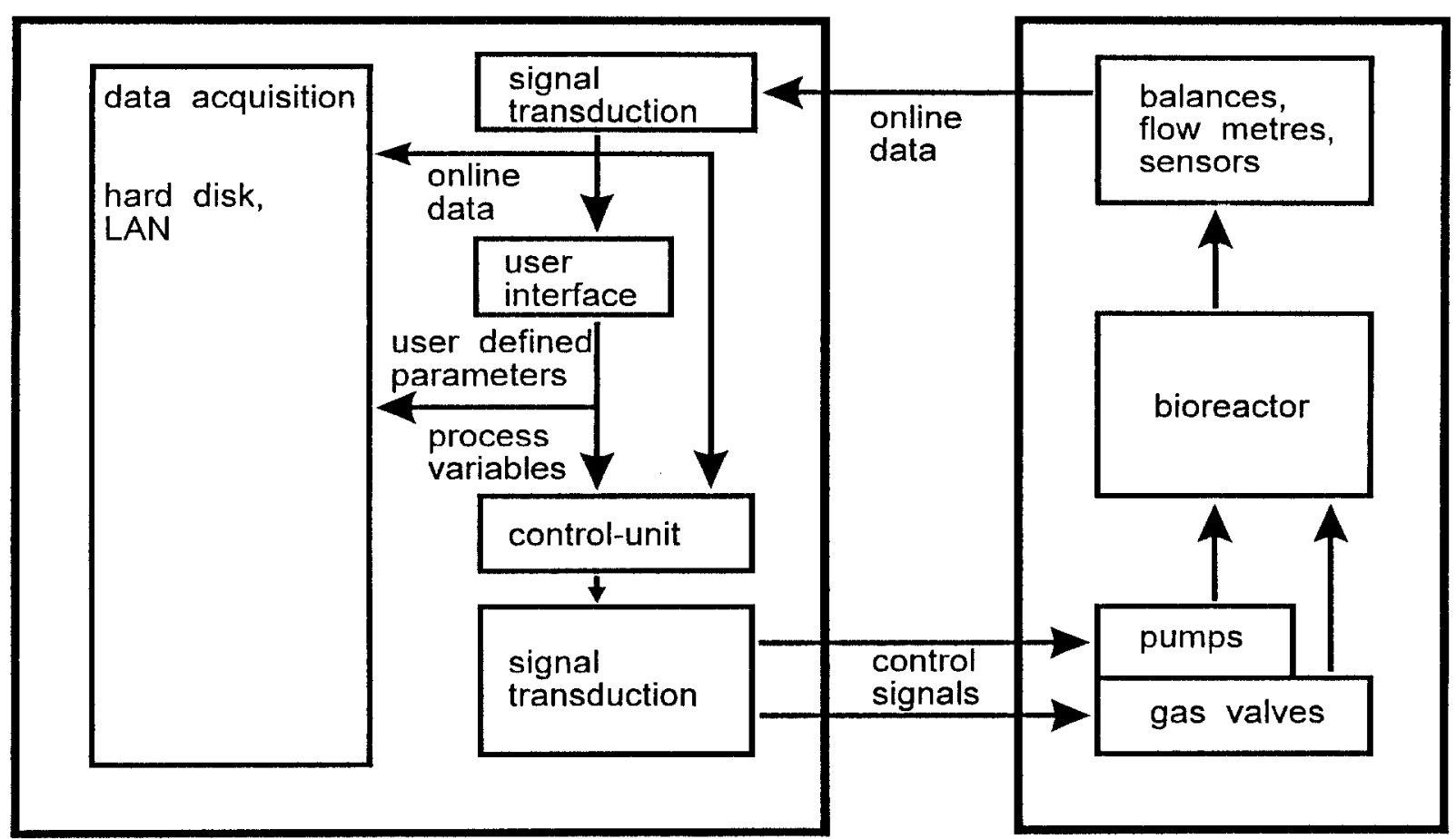

Data Acquisition and Control System CICS

Fermenter Setup

Fig. 1. The configuration of the computer integrated control system CICS is shown schematically.

Control units for pumps, aeration and data filing often are discrete modules. Manual control has to take place at different locations in the experimental setup and the medium flow rates are defined via revolutions per minute of the peristaltic pump rotor. Consequently, calculation and adjustment of feed, harvesting and dilution rates are very difficult and complicated. Since the amount of data accumulated during a continuous fermentation of 60 days and more may be voluminous, a suitable compression for on-line data storage is necessary to reduce the archive file length.

There is a tremendous need for a PCS, which facilitates the culture process handling by unifying data acquisition and control components.

\section{Material and methods}

\section{The CICS - data acquisition and control system}

The final configuration of the computer integrated control system (CICS), which is schematically shown in Figure 1, was developed as a compromise between the technical possibilies and the demands that will be made of this system. The primary features of the control setup include the following:

1. Central user interface (terminal/monitor); information about all process relevant values; allowing manual operations necessary during a running process.

2. Reception of on-line data like $\mathrm{pH}, \mathrm{pO}_{2}$, filling level of fermenter and medium vessels.

3. pH and oxygen partial pressure control by regulating the mass flows of air, oxygen, nitrogen and carbon dioxide.

4. Reception of actual gas flow, possible by using thermal mass flow controllers $\left(0-10 \mathrm{Nl} \mathrm{h}{ }^{-1}\right.$, HITEC, Ruurlo, NL).

5. Complete control of up to four pumps (WatsonMarlow 501 U, Watson-Marlow, Fallmouth, UK) dependant on the current fermentation mode.

6. Providing data collection and filing of on-line and used defined data.

\section{Hardware}

All on-line data $\left(\mathrm{pH}, \mathrm{pO}_{2}\right.$, balances and aeration) are connected to the computer integrated ADA (ADA 16, 


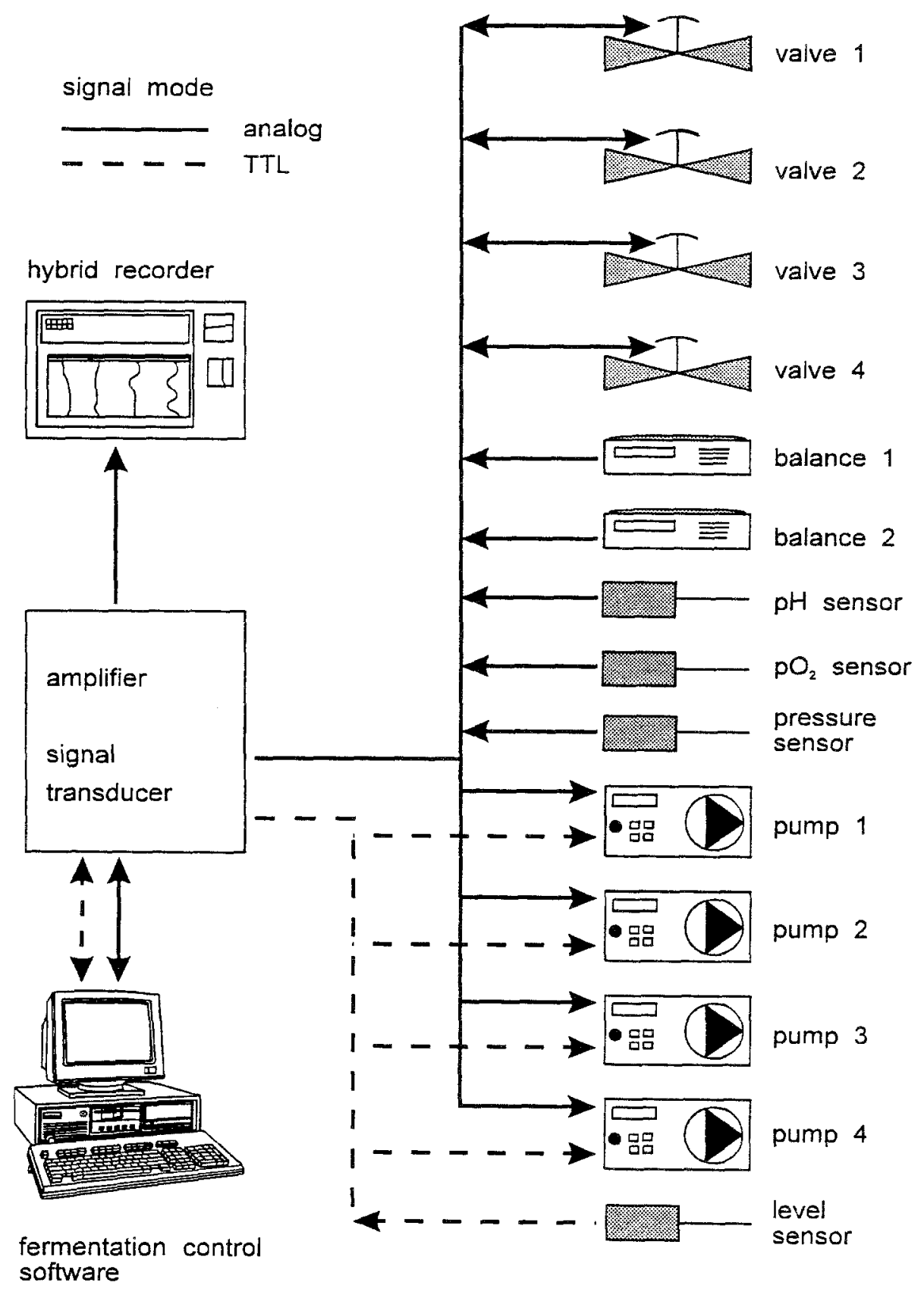

Fig. 2. Summary of possible hardware connections. Computer output signals are transduced, if required, and directly control the fermenter periphery. Input data are collected, transduced if necessary, processed and stored.

1989a+b) and TTL (Fischer, 1990) - interface cards (see Fig. 2). A transducer changes the voltage of this input signals into $0-2 \mathrm{~V}$ or $0-10 \mathrm{~V}$, respectively, needed for the interface cards. This renders a central processing of all on-line data from probes, and measuring devices like balances or mass flow controllers. If the voltage signals are compatible, the program can be adapted to various other sensors. The transduced output controls aeration valves, feeding and harvesting pumps.

Minimal requirement for running the process control system is an AT386 computer, $25 \mathrm{MHz}$, equipped with $2 \mathrm{MB}$ RAM and $20 \mathrm{MB}$ hard disk under a multitasking operation system like MultiUserDos 5.1 (Nov- 


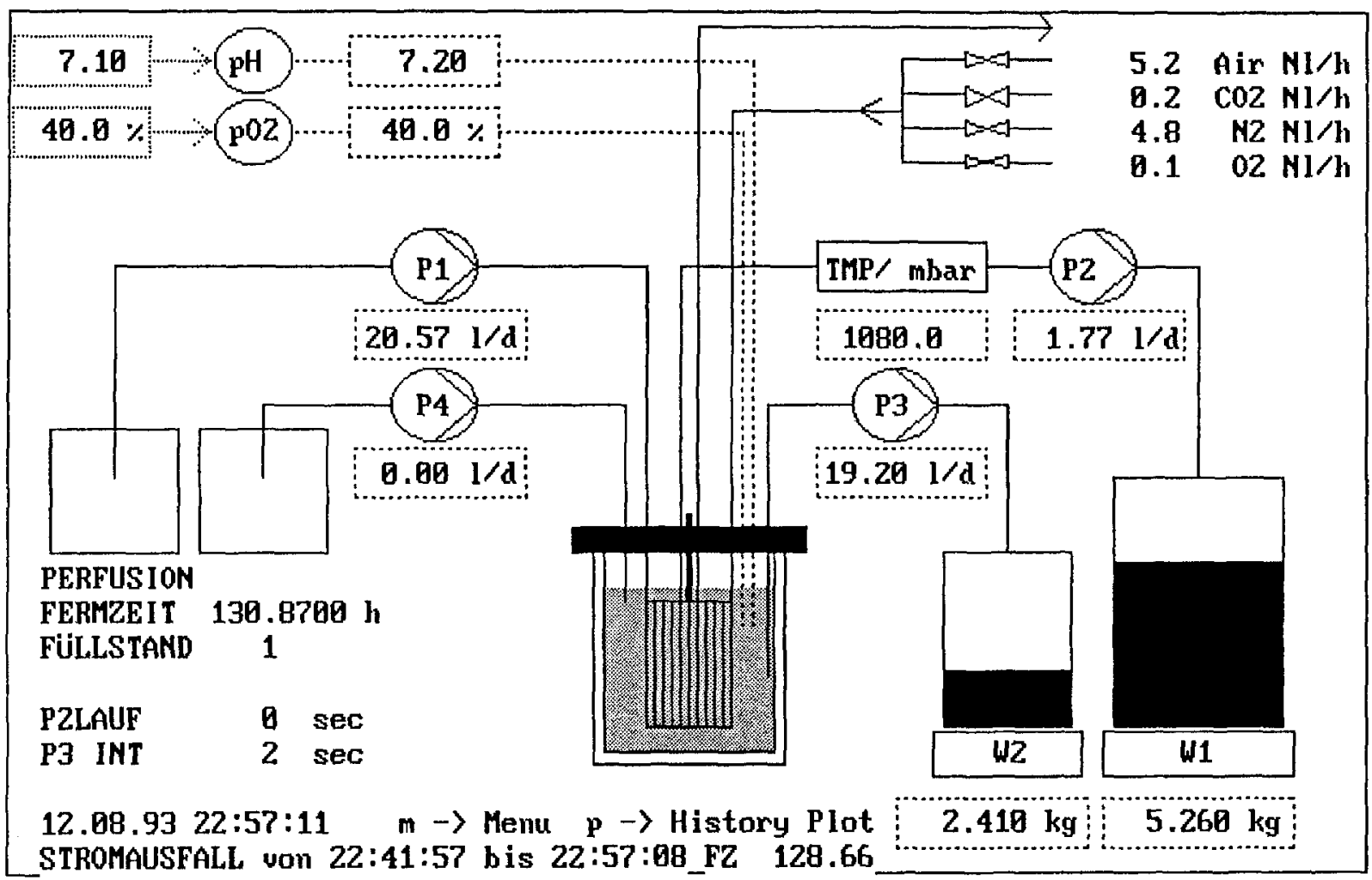

Fig. 3. Hardcopy of on-line main screen of CICS software.

ell) or OS/2 2.x (IBM, 5 MB RAM required). Connection to a computer network is possible and advantegeous.

\section{Software}

The central processing of all on-line and used defined data is done by a TurboBASIC compiled program. Controlling (ddc), data acquisition, data recording and user interface are totally integrated.

The status of the fermentation process is shown on a main screen (Fig. 3), which provides information about all relevant process and user defined variables like $\mathrm{pH}, \mathrm{pO}_{2}$, set points and flow rates of liquids and gases. The signal of the level sensor is shown directly: 1 - maximum level, 0 - level beyond maximum.

As far as data acquisition, pumps, controllers and aeration are concerned, all manual interventions during fermentation happen in menus at the terminal. Menus are as secure as possible against nonsense inputs. Senseless data like inputs that provoke a division by zero, pump rates that are higher than the maximum output or negative time intervals will be rejected.
A menu called 'fermentation mode' permits switching between batch, fed batch, chemostat and perfusion fermentation formats. After calibration of the peristaltic pumps at the beginning of each fermentation period, all relevant inputs are done in the units litre per day. A menu called 'pump control/pump parameters' provides a direct control device for the flow rates and intervals. The input of primary parameters like perfusion, harvest and dilution rate is possible in special control menus, offered for every fermentation mode. The control signals for the pumps are figured out automatically.

If required, a direct control of the gas flow controller is also possible. The corresponding menu allows set points between zero and the maximum mass flow for each valve.

During the fermentation $\mathrm{pH}$ and $\mathrm{pO}_{2}$ are controlled by software simulated PID controllers (ddc). Depending on the oxygen requirements of the culture the controller combines air and nitrogen or air and oxygen producing a constant $10 \mathrm{~N} 1 \mathrm{~h}^{-1}$ gas flow. Control of $\mathrm{pH}$ can take place supplementary with up to $10 \mathrm{Nl}$ $\mathrm{h}^{-1}$ carbon dioxide. Control parameters as set point, 


\section{$\mathrm{pO}_{2}$ [\%Air sat.]}

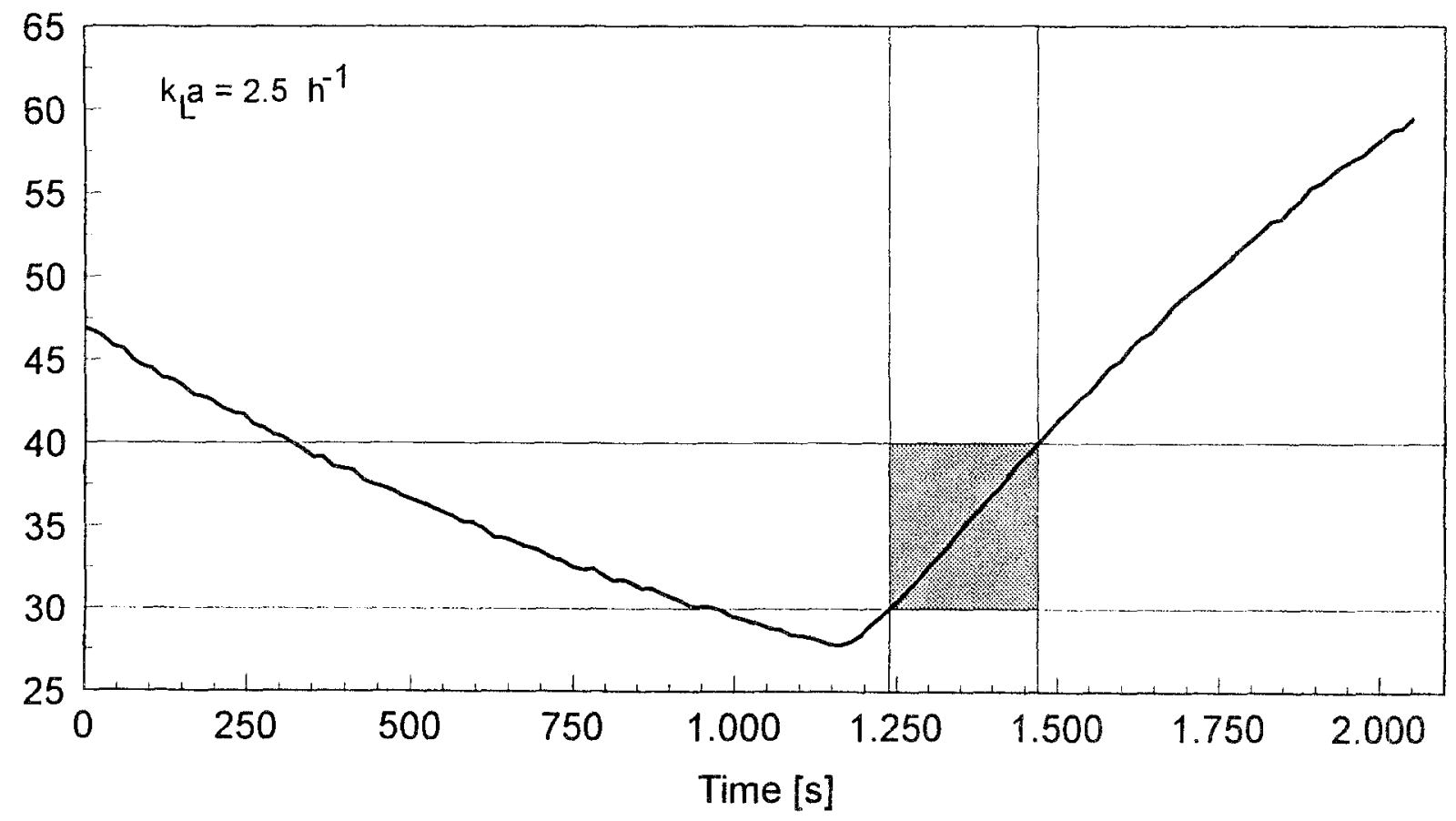

Fig. 4. Time course for $\mathrm{pO}_{2}$, generated by the PCS for the measurement of $\mathrm{k}_{\mathrm{L}}$ a-value. The $\mathrm{k}_{\mathrm{L}} \mathrm{a}$ is calculated automatically from the slope of the $\mathrm{pO}_{2}(\mathrm{t})$ curve in the range between 30 and $40 \%$ air saturation (marked box).

derivative and integration constant can easily be adapted to the current process conditions.

Data acquisition is realized in several files. The intervals between the single data points are use defined and can be changed every time during the fermentation. Each data set consists of every on-line measured value, date, time and fermentation duration.

The short term filing is done both in a RAM-matrix and in a short term archive file on hard disk, including the last 540 data sets. It can be displayed on screen as a 'history plot' and if the default value for the short term interval ( 60 seconds) is used the file will represent the last nine hours of fermentation.

A second, long term archive file consists of arithmetic mean values of the short term data received in the long term interval of the course of the whole fermentation. Both files are stored in a compressed format, which reduces the file length. A complete long term file, including twelve hundred data sets, needs only about $80 \mathrm{~KB}$ memory space on hard disk. This is equivalent to 30 days fermentation duration if the default value for the long term interval (60 minutes) is used. A third initialization file stores all current user defined data like control parameters, pump rates, tara weights of balances, calibration constants, etc. The usage of a computer network or a multitasking operating system provides a permanent grip on the stored data. After power or software failure, CICS is able to restart the fermentation in the most recent state by loading all required data from archive files. During the failure, no action will take place, because both PCS and fermenter setup are parts of the same circuit. The duration of software failures is at most one minute until the system works properly again. A main screen message shows time and duration of the power failure. After repeated error occurrence the system runs automatically in 'secure' batch mode to prevent pump malfunction.

Further on, the program provides standard operations like pump and balance calibration or OTR and $\mathrm{k}_{\mathrm{L}} \mathrm{a}$ value determination. The latter is done by the dynamic method (Vorlop 1990). For this the control program produces a $\mathrm{pO}_{2}$-profile like it is shown in Fig. 4.

All in all, results become more reproducible and the procedures cause no additional working effort. The accumulated data are stored in special files for later manipulation in word processing or spreadsheet software. 


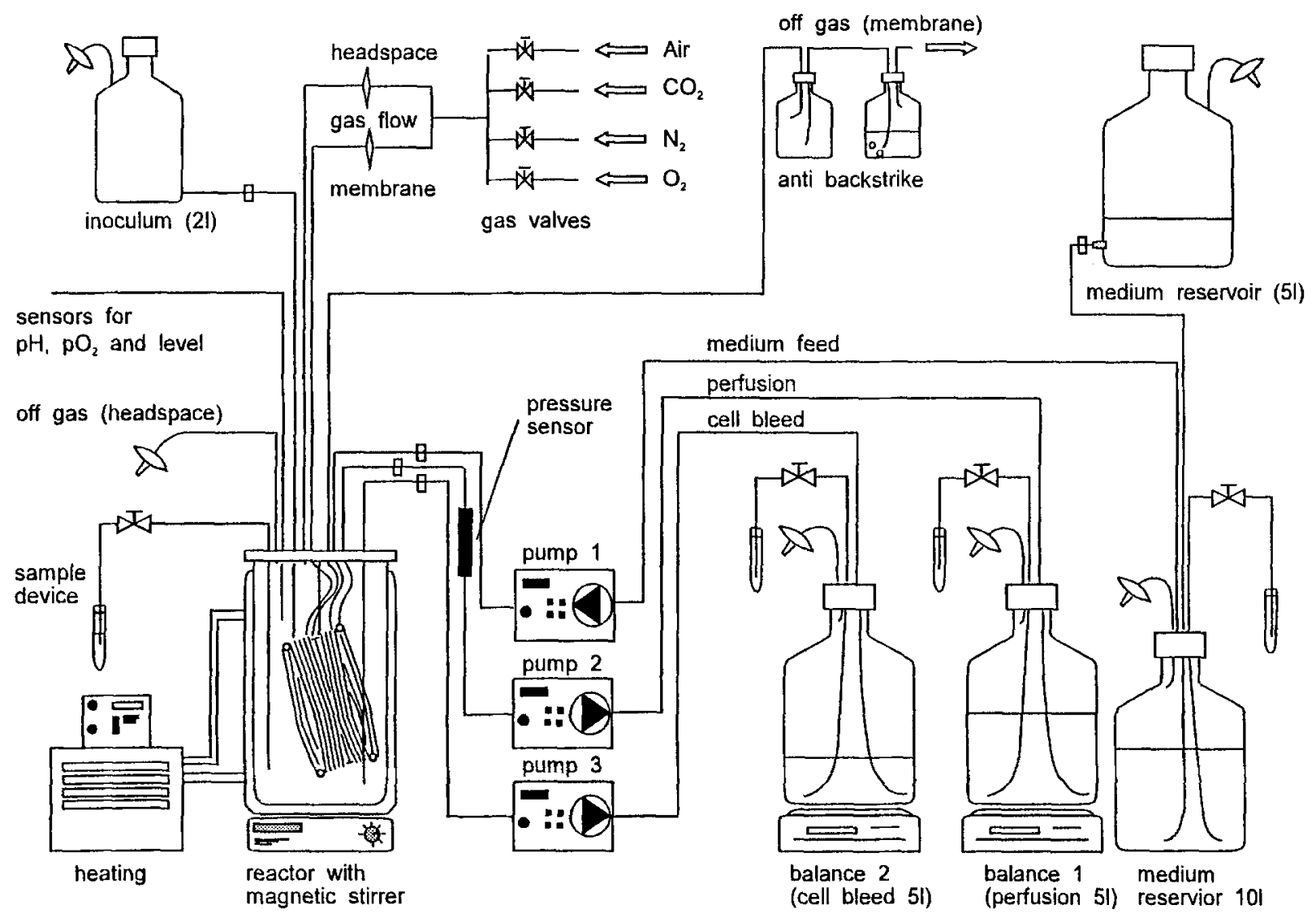

Fig. 5. Scheme of bioreactor and periphery setup controlled by CICS for the fermentation described in this study.

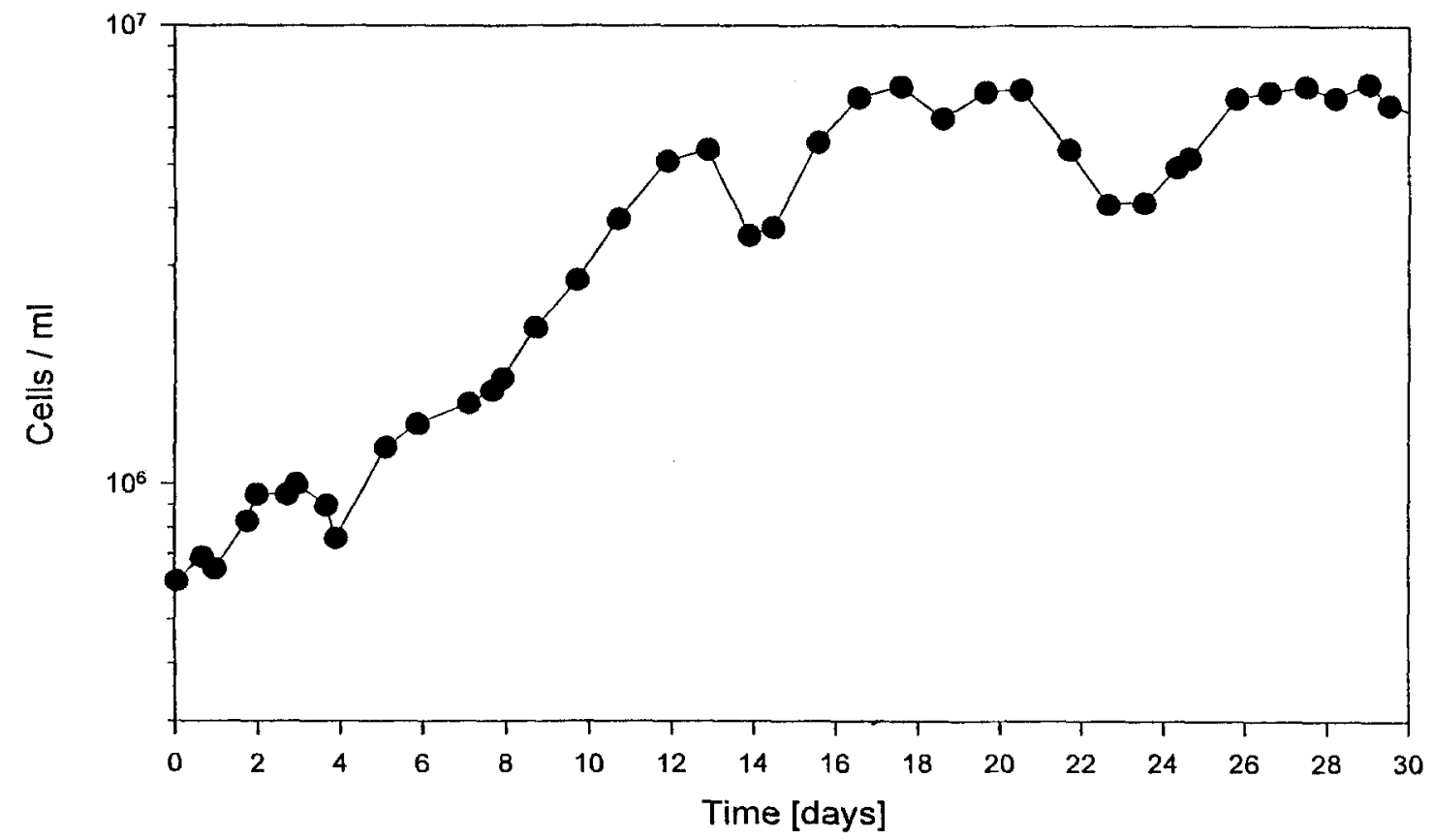

Fig. 6. Time course of total cell density of CHO fermentation. 


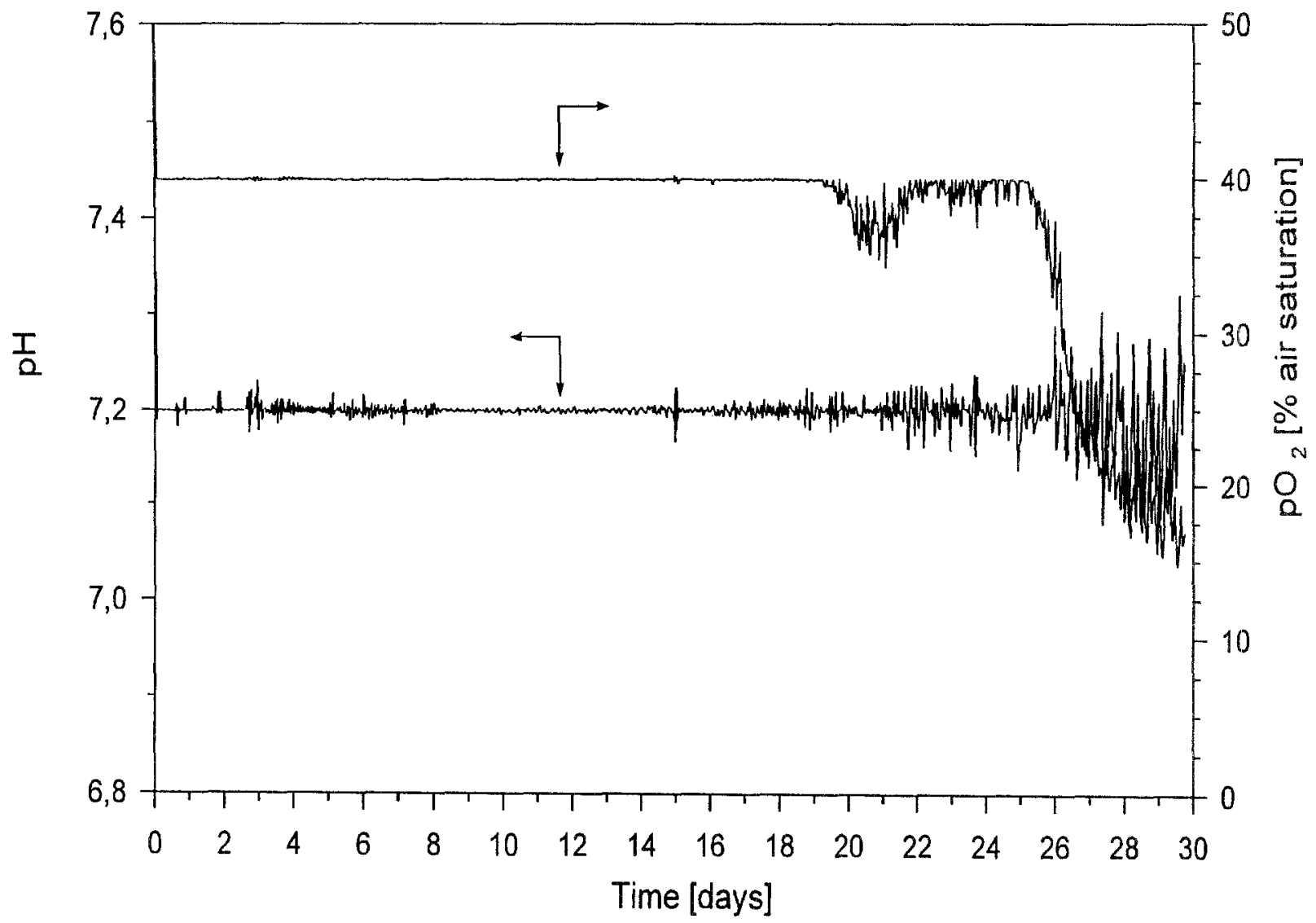

Fig. 7. History plot of $\mathrm{pH}$ and $\mathrm{pO}_{2}$ on-line data during $\mathrm{CHO}$ fermentation.

Reactor setup and cell culture

A fermentation of recombinant CHO cells (ZettlmeissI et al. 1987) (Behringwerke AG, Marburg, FRG) was carried out in a 2 L BIOSTAT BF2 bioreactor (B. Braun Biotech International, Melsungen, FRG) with bubblefree aeration (Lehmann et al., 1988) and internal cell retention via microporous membranes (Büntemeyer $e t$ al., 1987) (Fig. 5) (polypropylene for aeration, polysulfone for perfusion, Microdyn, Wuppertal, FRG). A serum-free, low protein medium DMEM/F12 1:1 (Gibco, Paisley, UK) as described in detail previously (Riese et al., 1993) was used. Counts of cell nuclei were figured out microscopically by crystal violet staining. A trypan blue stain allowed the determination of vital cells.

\section{Results}

The control software was installed on an AT386 computer, $25 \mathrm{MHz}$ with $5 \mathrm{MB}$ RAM under the multitasking operating system MultiUserDos 5.1 (Noveli) integrated in a local area network (Netware 386 V 3.11). All software features were adapted to the flow scheme shown in Fig. 5. Four gas mass flow controllers (Air, $\mathrm{O}_{2}, \mathrm{~N}_{2}, \mathrm{CO}_{2}$ ) were used to provide an appropriate gas mix at each state of the culture, depending on data from $\mathrm{pH}$ and $\mathrm{pO}_{2}$ sensors. Two pumps were controlled to perform a continuous perfusion mode with internal cell retention (microfiltration membrane) in a special manner to avoid early clogging of the membrane. Backflushing with fresh medium is integrated in this procedure (described in detail in Büntemeyer et al., 1987). Additionally, a third pump is controlled to carry out cell removal for maintaining steady state cell concentrations. Since CHO-cells form aggregates while growing in suspension culture, the cell bleed must be carried out discontinuously to prevent cell 


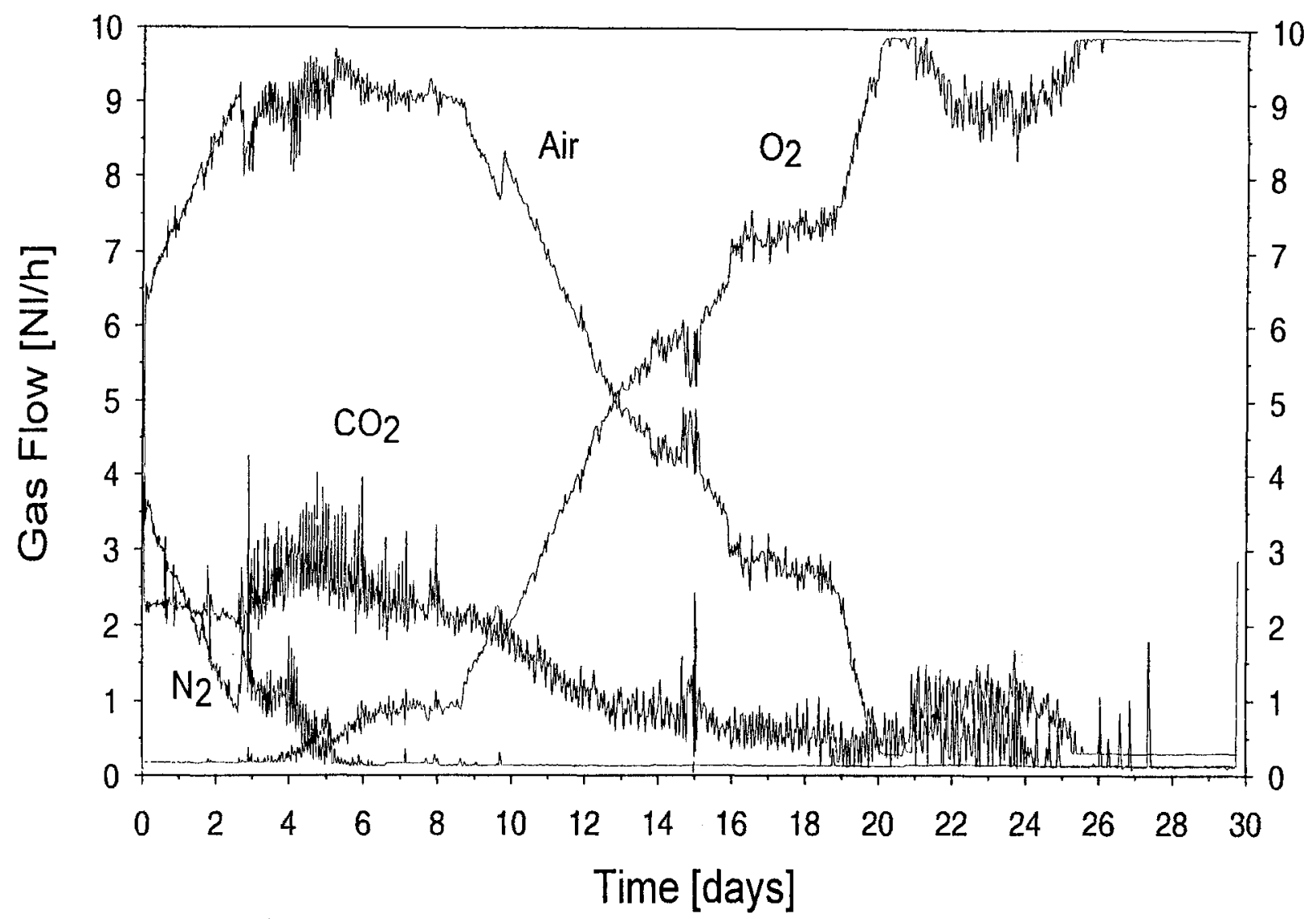

Fig. 8. History plot of the flow of the four controlled gases (air, $\mathrm{O}_{2}, \mathrm{~N}_{2}, \mathrm{CO}_{2}$ ) during CHO fermentation depending on $\mathrm{pH}$ and $\mathrm{pO} 2$ data.

sedimentation in the sampling tube. It is easy to adapt the control program in a way that the bleed pump works only for short intervals with high flow rates. The flow rates were calculated automatically after input of bleed rate and interval.

Data from two balances were also collected on-line to calculate actual flow rates for the verification of all pump rates. An on-line signal from a pressure sensor was used to monitor the fouling status of the perfusion membrane. In Fig. 6 the changes in cell concentration during a 30 day $\mathrm{CHO}$ cultivation are shown. After a four days batch, mode status was switched to perfusion mode. Additionally, the cell bleed stream was started at day 12 to maintain a stable cell density. The cells grew slowly (typical for this $\mathrm{CHO}$ cell line), reaching a final steady state density of about $8 \times 10^{6}$ total cells per ml. From Fig. 7 it can be seen that for the first 26 days both $\mathrm{pH}$ and $\mathrm{pO}_{2}$ could be kept very close to their set point values of $\mathrm{pH}=7.2$ and $\mathrm{pO}_{2}=40 \%$ air saturation, respectively. Only for the last four days $\mathrm{pO}_{2}$ could not be maintained at $40 \%$ air saturation and $\mathrm{pH}$ showed a higher fluctuation. This can be explained by following the oxygen gas flow in Fig. 8. The instability in $\mathrm{pO}_{2}$ and $\mathrm{pH}$ occurred when the oxygen valve is completely opened but the demand of the culture was not satisfied. In this case stable values of $\mathrm{pH}$ and $\mathrm{pO}_{2}$ could not be controlled sufficiently. The drop in $\mathrm{pO}_{2}$ beginning at day 26 is caused by a decrease in oxygen transfer capacity of the membrane aeration system due to membrane fouling effects of the hydrophobic polypropylene membrane. Higher oxygen transfer rates can be obtained by using a more hydrophobic membrane or by using a higher oxygen gas flow rate (requires a mass flow controller with more than a maximum of $10 \mathrm{Nl}$ $\mathrm{h}^{-1}$ ) or by employing a back pressure controller at the membrane outlet.

\section{Conclusion}

The presented 'computer integrated control system (CICS)' joins components of aeration and pump con- 
trol, data acquisition and central user interface for an easy to use and integrated detailed fermentation control system. In contrast to other PCS commonly used for small scale fermentations, like MICRO-MFCS (B. Braun International, Melsungen, FRG) or UBICON (EDS GmbH, Hannover, FRG) (Gollmer et al., 1992), this system was developed especially for application to cell culture bioreactors. Routine functions and interactive menus for the determination of parameters like dilution or bleed rate are embedded. Additionally user defined software controller for $\mathrm{pH}$ and $\mathrm{pO}_{2}$ are implemented in the process control program. Detailed data acquisition of all on-line data in user defined intervals is available in this system. A further processing of the data in spreadsheet programs is possible. Security features to overcome minor and major problems including power failure hardware and software errors are implemented in the program to guarantee correct fermentation performance and to minimize the loss of important cultures. An adaptation to all commercially available bioreactor systems, including amplifiers of sensors and probes, pumps and valves is easily possible if analog signals for data sampling and device control are available.

\section{Acknowledgements}

This work was partly supported by the project 'Development of a procedure and a plant for the recirculation of nutrient media for animal cell culture' (BMFT ref. no 0319346A) of the German Ministry of Science and Technology. We like to thank the Beringwerke AG,
Marburg, FRG, for kindly supplying the CHO cell line and Microdyn, Wuppertal, FRG, for supplying the polysulfone membrane.

\section{References}

ADA 16: 8-16 Bit AND-D/A-Wandlerkarte für PCs (Part 1 ELV Journal $2 / 89$ (1989a).

ADA 16: 8-16 Bit A/D-D/A-Wandlerkarte für PCs (Part 2 ELV Journal 3/89 (1989b).

Büntmeyer H, Bödeker BGD and Lehmann J (1987) Membranestirrer-reactor for bubble free aeration and perfusion. In: Modern Aproaches to Animal Cell Technology, Spier RE and Griffiths JB (eds), Butterworth, London, 411-419.

Fischer J, Werges U (1990) MSR-Karten für IBM-Kompatible. Elector, 9 (1990), 24.

Gollmer K, Gäbel T, Nothnagel J and Posten C (1992) UBICON a universal bioprocess control system. In: DECHEMA Biotechnology Conferences 5, VCH Verlagsgesellschaft, Weinheim.

Lehmann J, Vorlop J and Buintemeyer H (1988) Bubble free reactors and their development for continuous culture with cell recycle. In: Animal Cell Biotechnology 3, Spier RE and Griffiths JB (eds), Academic Press, London, 221-237.

Riese U, Heidemann R, Lütkemeyer D and Lehmann J (1993) Partial cell culture medium recirculation: Experience in pilot scale. In: Animal Cell Technology: Basic and Applied Aspects, Kaminogawa S, Ametani A and Hachimura S (eds), Kluwer, Dordrecht, 443-446.

Vorlop J (1990) Entwicklung eines Membranrührers zur Blasenfreien Begasung und Durchmischung von Zellkulturen im Pilotmaßstab. PhD Thesis, Technical University of Braunschweig.

Zettlmeissl G, Ragg H and KLarges HE (1987) Expression of biologically active human antithrombin III in Chinese hamster ovary cells. Biotechnology 5: 720-725.

Address for correspondence: Heino Büntmeyer, University of Bielefeld, Technical Faculty, Institute of Cell Culture Technology, P.O. Box 100131, 33501 Bielefeld, Germany. 